\title{
Numerical studies of confinement in the lattice Landau gauge
}

\author{
Hideo Nakajima $^{\mathrm{a}}$, Sadataka Furui ${ }^{\mathrm{b}}$ and Azusa Yamaguchi ${ }^{\mathrm{c}}$ \\ ${ }^{a}$ Department of Information Science, Utsunomiya University, \\ Utsunomiya 321-8585 Japan (e-mail nakajima@is.utsunomiya-u.ac.jp) \\ ${ }^{\mathrm{b}}$ School of Science and Engineering, Teikyo University, \\ Utsunomiya 320-8551, Japan (e-mail furui@liberal.umb.teikyo-u.ac.jp) \\ ${ }^{\mathrm{c}}$ Department of Physics, Ochanomizu University, \\ Tokyo 112-8610 Japan (e-mail: azusa@sokrates.phys.ocha.ac.jp).
}

Critical conjectures on confinement in the Landau gauge is numerically tested in focus to Gribov copy effects. One of the subjects is of the Kugo-Ojima confinement criterion and the other is of various viewpoints in the Gribov-Zwanziger theory. We use the smearing gauge as a reference gauge free of Gribov copy, and performed three types of simulations, $\log U, U$-linear and $\log U$ in the smearing gauge. It is found that Gribov copy effect on the Kugo-Ojima parameter is small. $\log U$ and $U$-linear simulations yield only global scale factor difference in gluon propagator and in ghost propagator, and about 10\% difference in Kugo-Ojima parameter. The horizon function defined by Zwanziger is evaluated in three types of gauge field and compared. All data show the negative horizon function as expected.

\section{INTRODUCTION}

Gribov pointed out that there exists a fundamental problem with respect to the field theoretic description of QCD in its quantization, due to gauge fixing degeneracy[1]. Gribov conjectured that the linear potential of quarks may be caused by an enhancement of the singularity of the ghost propagator due to the restiriction of the gauge field on the transverse plane. Zwanziger developed further the lattice gauge theory along the line of Gribov, and discussed its continuum limit [3].

Kugo ond Ojima developed the operator formalism of QCD starting from the standard BRST (Becchi-Rouet-Stora-Tyutin) symmetric Lagrangian, and derived Kugo-Ojima criterion, a sufficient condition for the colour confinement, i.e., the absence of free single coloured states in the asymptotic Hilbert space 2]. Even though there is no question in validity of the starting Lagrangian as the perturbation theory, the validity is unclear as the full theory, if one thinks of the Gribov problem which emerges in the 'large' amplitude region of the gauge field. There is a discussion of Fujikawa[5] and Hirschfeld[6] trying to justify the standard Lagrangian. This point is intimately related to a problem in numerical measurement[7] of gauge non-invariant quantity in presence of Gribov copy $[\mathbb{8}]$.

The Kugo-Ojima theory and Gribov-Zwanziger theory appear to stand on different footings, but each presents its own critical conjectures on confinement problems. It is meaningful to explore their validity numerically by lattice QCD dynamics. We test how numerically satisfied is the Kugo-Ojima criterion that a coefficient $u_{b}^{a}(0)$ in the two-point function produced by the ghost, the antighost and the gauge field becomes $-\delta_{a}^{b}$, and investigate how gluon propagator and ghost propagator behave in the infrared region. We also look at value of Zwanziger's horizon function to test if his horizon condition is satisfied 3]. The theoretical problem of quantization caused by the Gribov copy reflects as the Gribov noise problem in measurement of gauge non-invariant quantity, and it is favoured to choose a unique copy among others, i.e., a new gauge without Gribov copy 
(see [8] in more detail). As finding algorithms of unique gauge fixing is difficult in various gauges in general, random statistical method is commonly used, but it is cautioned that its performance is sensitive to incorporated algorithms in some cases [9]. Laplacian gauge fixing is well known as a unique gauge fixing, but only known method in Landau gauge is smearing gauge fixing $\llbracket$. We call this gauge as smearing gauge and adopt it as a reference gauge to investigate the Gribov copy effect.

There are some options of gauge field definition $A_{\mu}(U)$ in lattice gauge theory. We compare numerical data with respect to two types of definition, $\log U$ and $U$-linear.

\section{Gauge fields and lattice Landau gauge}

Options of $A_{\mu}(U)$ for $S U(3)$ lattice QCD are

1. $\log U ; U_{x, \mu}=e^{A_{x, \mu}}, A_{x, \mu}^{\dagger}=-A_{x, \mu}$, where |eigenvalue of $A_{x, \mu} \mid \leq 4 \pi / 3[10$.

2. $U$-linear; $A_{x, \mu}=\left.\frac{1}{2}\left(U_{x, \mu}-U_{x, \mu}^{\dagger}\right)\right|_{t r l}$. 33 .

In both cases the Landau gauge, $\partial A^{g}=0$, can be characterized in use of optimizing functions $F_{U}(g)$ of $g$, such that $\delta F_{U}(g)=0$ for any $\delta g$, respectively; (trl means the traceless part)

$$
\begin{aligned}
& \text { 1. } F_{U}(g)=\left\|A^{g}\right\|^{2}=\sum_{x, \mu} \operatorname{tr}\left(A_{x, \mu}^{g} A_{x, \mu}^{g}\right), \\
& \text { 2. } F_{U}(g)=\sum_{x, \mu}\left(1-\frac{1}{3} \operatorname{Retr} U_{x, \mu}^{g}\right) .
\end{aligned}
$$

In both options, the variation of the optimizing function, $F_{U}(g)$, under infinitesimal gauge transformation $g^{-1} \delta g=\epsilon$, reads as

$\Delta F_{U}(g)=-2\left\langle\partial A^{g} \mid \epsilon\right\rangle+\left\langle\epsilon\left|-\partial D\left(A^{g}\right)\right| \epsilon\right\rangle+\cdots$,

where $D_{\mu}(A)$ denotes the covariant derivative in each definition respectively; in short notations,

$U=U_{x, \mu}, A=A_{x, \mu}$, and $\partial_{\mu} \phi=\phi(x+\mu)-\phi(x)$, $\bar{\phi}=\frac{1}{2}(\phi(x+\mu)+\phi(x))$,

$$
\begin{aligned}
& \text { 1. } D_{\mu}(A) \phi=S(\mathcal{A}) \partial_{\mu} \phi+[A, \bar{\phi}] \text {, } \\
& \text { where } \mathcal{A}=\operatorname{adj}_{A}=[A, \cdot], S(x)=\frac{x / 2}{\operatorname{th}(x / 2)} \text {. } \\
& \text { 2. } D_{\mu}(U) \phi=\left.\frac{1}{2}\left\{\frac{U+U^{\dagger}}{2}, \partial_{\mu} \phi\right\}\right|_{t r l .}+[A, \bar{\phi}] .(3)
\end{aligned}
$$

\section{The Kugo-Ojima confinement criterion and the Gribov-Zwanziger's theory}

\subsection{The Kugo-Ojima criterion $[2]$}

The Kugo-Ojima criterion is stated as follows; $u^{a b}(0)=-\delta^{a b}$, where $u^{a b}$ is defined by the following two-point function as,

$$
\begin{aligned}
& \left(\delta_{\mu \nu}-\frac{p_{\mu} p_{\nu}}{p^{2}}\right) u^{a b}\left(p^{2}\right) \\
& =\frac{1}{V} \sum_{x, y} e^{-i p(x-y)}\left\langle\operatorname{tr}\left(\lambda^{a \dagger} D_{\mu} \frac{1}{-\partial D}\left[A_{\nu}, \lambda^{b}\right]\right)_{x y}\right\rangle(4)
\end{aligned}
$$

where angle brackets denote sample average, and $\lambda^{a}$ is a normalized $\left(\operatorname{tr} \lambda^{a \dagger} \lambda^{b}=\delta^{a b}\right)$ antihermitian basis of Lie algebra, $V$ a lattice volume.

\subsection{Zwanziger's theory [3]}

Zwanziger classified transverse space of gauge field into some sets. $F_{U}(g)$ takes the local minimum in the Gribov region $\Omega$, and the global minimum in the fundamental modular region $\Lambda$, $\Lambda \subset \Omega$. Within $\Lambda$, there exists no gauge fixing degeneracy up to global gauge transformation. We call this gauge $\Lambda$ gauge. For purpose of gauging away Bloch wave states, Zwanziger narrowed down $\Lambda$ to core region $\Xi \subset \Lambda$ : Let the space of configuration of period $L$ be $\Pi_{L}$, and the fundamental modular region in the $\Pi_{L}$ be $\Lambda_{L}$, and let $\Lambda_{L}^{N}=\Lambda_{N L} \cap \Pi_{L}$, then the core region is given by $\Xi_{L} \equiv \Lambda_{L}^{\infty}$. Note, however, that by this definition, considerably large amount of points in $\Lambda_{L}$ fail to come into $\Lambda_{L}^{N}$ not because they can be gauge transformed to the point in $\Lambda_{L}^{N}$, but because they fail to satisfy $L$-periodicity condition when gauge transformed to $\Lambda_{N L}$. From this view point, narrowing down to the core region looks like a kind of artificial selection of configurations. Thus Zwanziger's basic hypothesis is that partition functions defined by path integrals over $\Xi_{L}$ and $\Lambda_{L}$, give the same limit, $\lim _{L \rightarrow \infty} Z_{\Xi_{L}}=\lim _{L \rightarrow \infty} Z_{\Lambda_{L}}$. Since this hypothesis is highly dynamical, it is to be checked somehow, e.g., by simulation. One can show that the following horizon function $H(U)$ is negative in the above defined core region $\Xi$ by estimating increase of $F_{U}(g)$ by Bloch wave excitation. $H(U)$ 
is given as follows;

$$
\begin{aligned}
& H(U)=\sum_{x, y, a} G_{\mu \mu x y}^{a a}-8 E(U), \\
& G_{\mu \nu x y}^{a b}=\operatorname{tr}\left(\lambda^{a \dagger} D_{\mu} \frac{1}{-\partial D}\left(-D_{\nu}\right) \lambda^{b}\right)_{x y},
\end{aligned}
$$

with

$$
\begin{aligned}
& \text { 1. } E(U)=\frac{1}{8} \sum_{l, a} \operatorname{tr}\left(\lambda^{a \dagger} S\left(\mathcal{A}_{l}\right) \lambda^{a}\right), \\
& \text { 2. } E(U)=\sum_{l} \frac{1}{3} \operatorname{Re} \operatorname{tr} U_{l},
\end{aligned}
$$

Statistical average defines a tensor $G_{\mu \nu x y}$ as $\left\langle G_{\mu \nu x y}^{a b}\right\rangle=G_{\mu \nu x y} \delta^{a b}$, provided colour symmetry is not broken. The Fourier transform of the tensor $G_{\mu \nu x y}$ takes a form

$G_{\mu \nu}(p) \delta^{a b}=\left(\frac{e}{4}\right) \frac{p_{\mu} p_{\nu}}{p^{2}} \delta^{a b}-\left(\delta_{\mu \nu}-\frac{p_{\mu} p_{\nu}}{p^{2}}\right) u^{a b},(9)$

where $e=\langle E(U)\rangle / V$. It is related with the horizon function as

$$
\frac{\langle H(U)\rangle}{V}=8\left[\lim _{p \rightarrow 0} G_{\mu \mu}(p)-e\right] .
$$

Note that $e=4$ if all link $U_{l}=1$. For purpose of path integral estimation of the partition function, a set augmented core region $\Psi=\{U: H(U) \leq 0\} \cap \Omega(\Xi \subset \Psi \subset \Omega)$ is defined, and from estimation of $Z_{\Psi}$, one derives the infinite volume limit 3 that $\lim _{V \rightarrow \infty} \frac{\langle H(U)\rangle}{V}=0$ which is called horizon condition. Putting KugoOjima parameter as $u^{a b}(0)=-\delta^{a b} c$, one finds from (9), (10), that the horizon condition is written as $\left(\frac{e}{4}\right)+3 c-e=3\left(c-\frac{e}{4}\right)=0$.

\section{Method of Landau gauge fixing, numer- ical results and discussion}

Our simulation was done for $\beta=6.0$ in lattice size $16^{4}$. Simulation of gauge fixed theory can be simply executed by gauge transforming a Boltzmann sample to the gauge, and measuring a quantity in question[7. This algorithm is unambiguous and correct only if the gauge is free of Gribov copy, or the quantity is gauge invariant. It is easily understood that if the quantity is gauge non-invariant and if there exists Gribov copy, numerical results depend not only on the gauge itself, but also on the algorithm employed which brings a sample to a gauge copy[9]. We performed three types of Landau gauge calculations, combinations of gauge field definitions and gauge fixing algorithms.

1. Gauge fixing with $\log U$ option using a direct Newton method, i.e., gauge transformation phase $\phi$ being given by $\phi=$ $(-\partial D(A))^{-1} \partial A[10]$. Gribov copy exists.

2. Gauge fixing with $\log U$ option using the smearing gauge fixing [4]. This smearing gauge is a unique Landau gauge. But our execution shows that the smearing gauge at $\beta=6,16^{4}$ does not coincide completely with $\Lambda$ gauge.

3. Gauge fixing with $U$-linear option using the non-stochastic-over-relaxation method after the process 1 . Gribov copy exists.

In our smearing gauge fixing, accuracy of plaquette value $E_{p}$ of smeared vacuum configuration is set as $1-E_{p}<10^{-7}$, since there appear a long plateau around $1-E_{p} \sim 10^{-4}$ in smearing process for many samples, and the finally obtained gauge fixed configuration depends critically on the situation if the smearing is finished before or after the long plateau.

Kugo-Ojima parameter is found to be $u_{b}^{a}(0) \sim$ -0.65 for cases 1,2 . and there is no remarkable difference. The magnitude of the parameter is about $10 \%$ less in case $3 . u_{b}^{a}$ does not look like tending to -1 up to the lattice size $16^{4}$. The reason is unclear so far. And Table 1 shows the KugoOjima parameters and values related to the horizon function. All values indicate negative horizon function 12.

Gluon propagator is infrared finite, and $\log -\log$ plot of unrenormalized gluon propagator is given in Fig 1, and about $20 \%$ difference of case 3 appear as a global scale factor, i.e., wave function renormalization, and this situation agrees with the discussion in the literature 11. Case 2 is almost same as case 1 . 
Table 1

Results of the Kugo-Ojima parameter $c$, 'trace' $e$ divided by the dimension 4 , and $h=c-e / 4$.

\begin{tabular}{llll}
\hline gauge fixing & $c$ & $e / 4$ & $h$ \\
\hline $\log U$ & $0.628(94)$ & $0.943(1)$ & -0.32 \\
smeared $\log U$ & $0.647(101)$ & $0.943(1)$ & -0.30 \\
$U$-linear & $0.576(79)$ & $0.860(1)$ & -0.28 \\
\hline
\end{tabular}

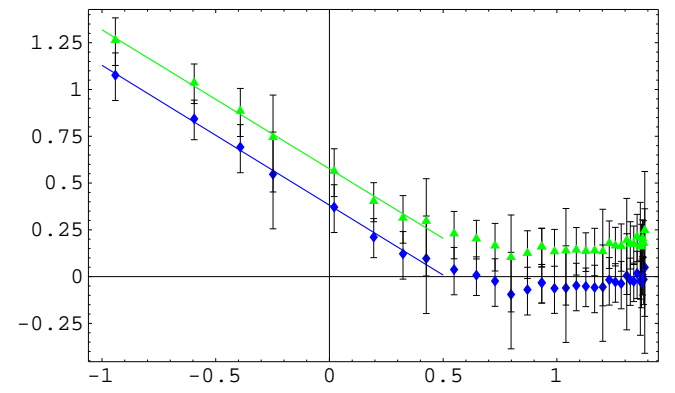

Figure 1. The log of the unrenormalized gluon propagator multiplied by $(q a)^{2}$ as a function of the $\log q a$ where $q$ is the lattice momentum. Triangles and the fitted line $-0.743 \log q a+0.576$ are in the $\log U$, and diamonds and the fitted line $-0.747 \log q a+0.382$ are in the $U$-linear.

$\log -\log$ plot of ghost propagator is given in Fig 2. The difference of case 1 and case 3 is the global scale factor as in the case of gluon propagator. Case 2 is almost same as case 1.

This work was supported by KEK Supercomputer Project(No.00-57), and JSPS, Grant-in-aid for Scientific Research(C) (No.11640251).

\section{REFERENCES}

1. V.N. Gribov, Nucl. Phys. B139, 1(1978).

2. T. Kugo and I. Ojima, Prog. Theor. Phys. Supp. 66, 1 (1979).

3. D. Zwanziger, Nucl. Phys. B412, 657 (1994).

4. J.E. Hetrick and P.H. de Forcrand, Nucl.

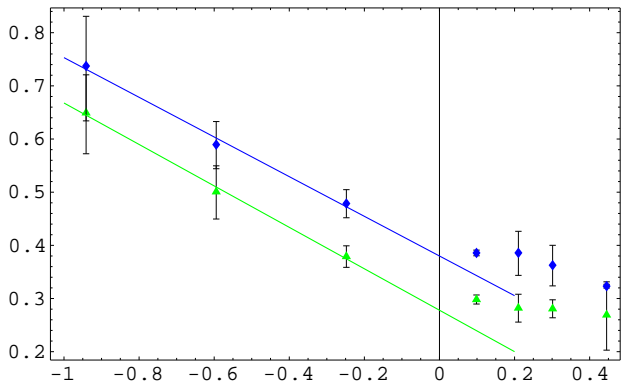

Figure 2. The log of the unrenormalized ghost propagator multiplied by $(q a)^{2}$ as a function of the $\log q a$ where $q$ is the lattice momentum. Triangles and diamonds are the same as Fig1. The fitted line is $-0.390 \log q a+0.278$ in the $\log U$ and $-0.373 \log q a+0.380$ in the $U$-linear.

Phys. B(Proc. Suppl.) 63A-C, 838 (1998).

5. K. Fujikawa, Prog. Theor. Phys. 61, 627 (1979).

6. P. Hirschfeld, Nucl. Phys. B157, 37 (1979).

7. J.E. Mandula and M. Ogilvie, Phys. Lett. B185, 127 (1987).

8. H.Nakajima, S.Furui, A.Yamaguchi, ICHEP 2000 contribution paper, hep-lat/0007001.

9. V.G. Bornyakov, D.A. Komarov, M.I. Polikarpov, hep-lat/0009035.

10. H.Nakajima and S. Furui, Nucl. Phys. B(Proc Suppl.)63A-C, 635,865 (1999); idem, Nucl. Phys. B(Proc Suppl.)83-84, 521 (2000); Confinement III proc., hep-lat/9809078; QNP2000 proc., hep-lat/0004023. 
11. L. Giusti, M.L. Paciello, S. Petrarca, B. Taglienti and M. Testa, hep-lat/9803021.

12. The value $e / 4$ in $\log U$ reported elsewhere so far was erroneous. 